\title{
Analysis of Gelatin Adulteration in Edible Bird's Nest using Fourier Transform Infrared (FTIR) Spectroscopy
}

\author{
Nurul H. Jamalludin", Nur A.Tukiran ${ }^{*}$ \\ \# Department of Biotechnology, Kulliyyah of Science, International Islamic University Malaysia (IIUM), IIUM Kuantan, \\ 25200 Kuantan, Malaysia \\ E-mail: hidayah.jamalluddin@gmail.com \\ * International Institute for Halal Research and Training (INHART), Level 3, KICT Building, International Islamic University Malaysia \\ (IIUM), Jalan Gombak, 53100, Selangor, Malaysia \\ Corresponding author e-mail: aziratukiran@iium.edu.my
}

\begin{abstract}
Fraudulent incorporation of cheaper materials such as porcine gelatin into edible bird's nest (EBN) can evolve into a problem for reasons related to religious, allergy, ethical, and legal requirement. Thus, this study aimed to detect porcine gelatin in the processed EBNs by using a combination method of Fourier transform infrared (FTIR) and chemometrics analysis, Principal Component Analysis (PCA). The use of FTIR spectroscopy in food analysis is becomes more attractive because of its cost-effective nature, nondestructive measurements as well as convenience for screening purposes. This method has been established to be useful for adulteration detection and quantification in various food products. However, its application as a sole method is often not reliable as some transitions of the spectrums are very complex or weak, making evaluation difficult. Thus, in this study FTIR data were further analysed with the chemometrics analysis. By considering all the data obtained, chemometrics makes better results feasible. Porcine gelatin exhibited a dominant band at Amide I indicating the adulteration of EBNs with porcine gelatin. The FTIR spectra were analysed using PCA in order to identify the adulteration percentage in the samples. In the assessment of the spiked samples, this method could detect at the minimum of $5 \%$ of porcine gelatin in EBNs. This method would be advantageous for ensuring quality of the EBN products in the market.
\end{abstract}

Keywords - Edible Bird's Nest (EBN); Fourier Transform Infrared (FTIR) spectroscopy; gelatin; Principle Component Analysis (PCA); Halal.

\section{INTRODUCTION}

Fraudulent incorporation of cheaper materials such as porcine gelatin into Edible Bird's Nest (EBN) can evolve into a problem for reasons related to religious, allergy, ethical, and legal requirement. Particularly, porcine is haram for Muslim hence adulteration of EBN with this material renders the product to be non-halal. Taking into account of low-priced and similarity in color and texture, porcine gelatin is a potential source of adulteration. In light of these concerns, it is of great significance to develop a reliable analytical method for EBNs authentication. Several analytical methods such as the sodium dodecyl sulfatepolyacrylamide gel electrophoresis (SDS-PAGE) [1], 2D SDS-PAGE [2], polymerase chain reaction (PCR) [2],[3], high performance liquid chromatography (HPLC) [4] and enzyme-linked immunosorbent assay (ELISA) [5],[6] have been developed. These methods demonstrated favorable establishment of adulterants detection, yet relied on expensive instruments and well-trained staff.

Alternatively, the use of Fourier Transform Infrared (FTIR) spectroscopy in food analysis is becomes more attractive because of its cost-effective nature, nondestructive measurements as well as convenience for screening purposes. This method has been established to be useful for adulteration detection and quantification in various food products such as sibutramine in tea and coffee [7], syrup in fruit nectars [8], and vegetable oils in olive oil [9]. However, its application as a sole method is often not reliable as some transitions of the spectrums are very complex or weak, making evaluation difficult. Currently, a raising interest has been shown in combining FTIR data with chemometric analysis. By considering all the data obtained, chemometrics makes better results feasible. Therefore, the aim of this study was to detect porcine gelatin in the processed EBNs using a combination of Fourier transform infrared (FTIR) and chemometrics analysis, Principal Component Analysis 
(PCA). Such assay would be advantageous for ensuring quality of the EBN products in the market.

\section{MATERIAL AND METHOD}

\section{A. Materials}

Two types of processed EBNs were obtained from traditional chinese medicine shop in Selangor, Malaysia. The sample included of cave nest (Aerodramus sp.) (CN) and house nest (Collocalia sp.)/ orange nest (ON) (Fig. 1). Seven samples of raw gelatins are tabulated in Table 1. Ultra-pure deionized water was used as buffer.

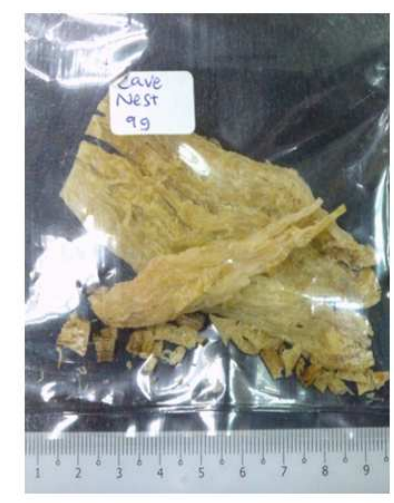

(a)

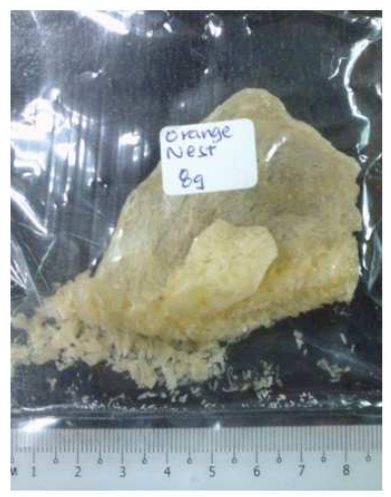

(b)
Fig.1 (a) Cave nest (Aerodramus sp.) (CN) and (b) house nest (Collocalia sp.)/ orange nest (ON).

TABLE I

SAMPLES OF RAW GELATIN

\begin{tabular}{|c|c|c|c|l|}
\hline No. & Species & Tissue & Type & Company name \\
\hline 1 & Porcine & Skin & A & $\begin{array}{l}\text { Sigma-Aldrich (St. Louis, } \\
\text { MO, USA) }\end{array}$ \\
\hline 2 & Porcine & Skin & A & $\begin{array}{l}\text { Restar Gelatin (Xiamen) Co. } \\
\text { Ltd. (China) }\end{array}$ \\
\hline 3 & Bovine & Skin & B & $\begin{array}{l}\text { Restar Gelatin (Xiamen) Co. } \\
\text { Ltd. (China) }\end{array}$ \\
\hline 4 & Bovine & Skin & A & $\begin{array}{l}\text { PB Gelatins (Vilvoorde, } \\
\text { Belgium) }\end{array}$ \\
\hline 5 & Bovine & Bone & B & $\begin{array}{l}\text { Halalgel Sdn. Bhd., } \\
\text { (Malaysia) }\end{array}$ \\
\hline 6 & Bovine & Skin & A & $\begin{array}{l}\text { Restar Gelatin (Xiamen) Co. } \\
\text { Ltd. (China) }\end{array}$ \\
\hline 7 & Fish & Skin & A & $\begin{array}{l}\text { Sigma-Aldrich (St. Louis, } \\
\text { MO, USA) }\end{array}$ \\
\hline
\end{tabular}

\section{B. Preparation of Spiked Samples}

Porcine gelatin was included in EBN samples to obtain spiked 5, 10, 15 and $20 \%(\mathrm{w} / \mathrm{w})$. The samples were subjected for FTIR analysis. Non-spiked samples were used as negative controls. Two set of samples have been set up for each treatment.

\section{FTIR}

The spectra was measured by using spectrometer, model Nicolet 6700 (Thermo Nicolet Corp., Madison, WI) that associated with the operating system of OMNIC (Version 7.0 Thermo Nicolet). Approximately $100 \mu \mathrm{L}$ of sample (10\% w/v) was deposited and spread uniformly using the tip of the micropipette onto the surface of attenuated total reflectance (ATR). The measurement was obtained in a range of 800$4000 \mathrm{~cm}^{-1}$. Two reproduce spectra were gathered for each sample.

\section{Statistical Analysis}

The quality comparison of FTIR spectrum was evaluated using the Principal Component Analysis (PCA), Unscrambler 9.7 (Camo, USA). Sixty locales of range were set as the variables. The score plot was obtained from the $\mathrm{PC} 1$ and PC2 as they contributed to the most extreme number of variability in the data input.

\section{RESULTS AND DISCUSSION}

\section{A. FTIR Spectra of Gelatins}

Gelatin comes from the partial hydrolysis of collagen. Generally, gelatin manufacturing processes comprising of three main stages which is pre-treatment of the raw material such as bone or skin, extraction of, and purification and drying.

Two gelatin types are therefore available, contingent on the pre-treatment process; (i) type A gelatin is obtained from acid-treated collagen suitable for less covalent cross - linked collagen in the pig or fish skin responsible for isoelectric point $\mathrm{pH} \sim 8-9$; (ii) gelatin type $\mathrm{B}$ is obtained from alkalitreated collagen and suitable for more complex collagen in bovine skins resulting from isoelectric point $\mathrm{pH} \sim 4-5$. Manufacture of gelatin from pig skin is limited to acid treatment as alkali processing prompts saponification of the fat substance, making further handling troublesome. The type and source of collagen will effect the characteristics of the resulting gelatins.

Generally, gelatins from porcine, bovine and fish exhibited very similar spectra. Three regions included are 1240-670 cm-1 (Amide III), 1560-1335 cm-1 (Amide II) and 1600-1700 cm-1 (Amide I). These results agree with those of Hashim et al. (2010) [10]. From the locations and intensities of the infrared bands, gelatin exhibited a dominant band at Amide I, which is generally assigned to an $\alpha$-helix structure [11]. The ruggedness of the spectra between Amide II and III was more apparent in fish gelatin compared to that in the porcine and bovine gelatin (Fig.2).

\section{B. Evaluation of Spiked Samples}

Figure 2 shows a comparison of spectra of spiked samples. The spectra exhibited that the FTIR is able distinguish between pure and adulterated EBNs. The spectra sort in ascending order according to the concentrations level of adulterant. The $\mathrm{CN}$ and $\mathrm{ON}$ nests exhibited similar pattern of spectra indicating the presence of similar compounds. The compounds namely hydroxyl, alkanes, alkyne, amide, amine, alkane, ether and alkene were identified [12]. The FTIR spectra were then analyzed using PCA in order to identify the adulteration percentage in the samples. 


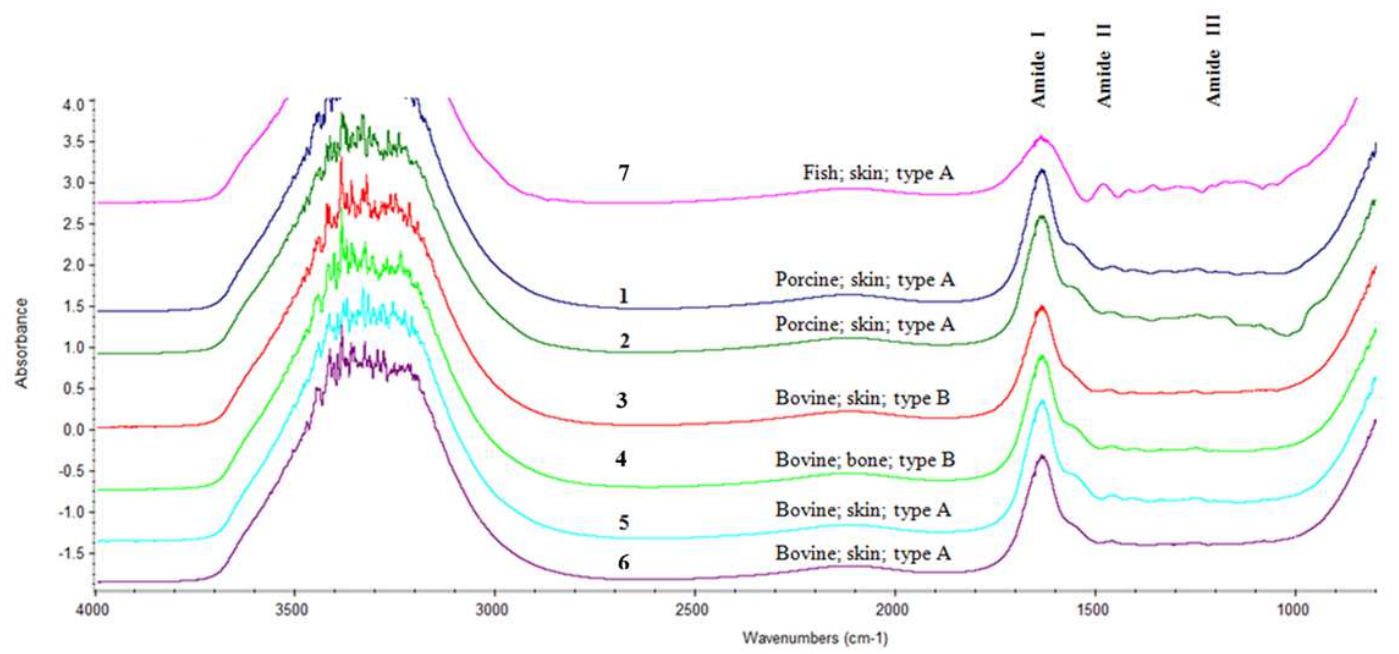

Fig. 2 FTIR spectra of raw gelatins. Number represented the sample number in Table 1.

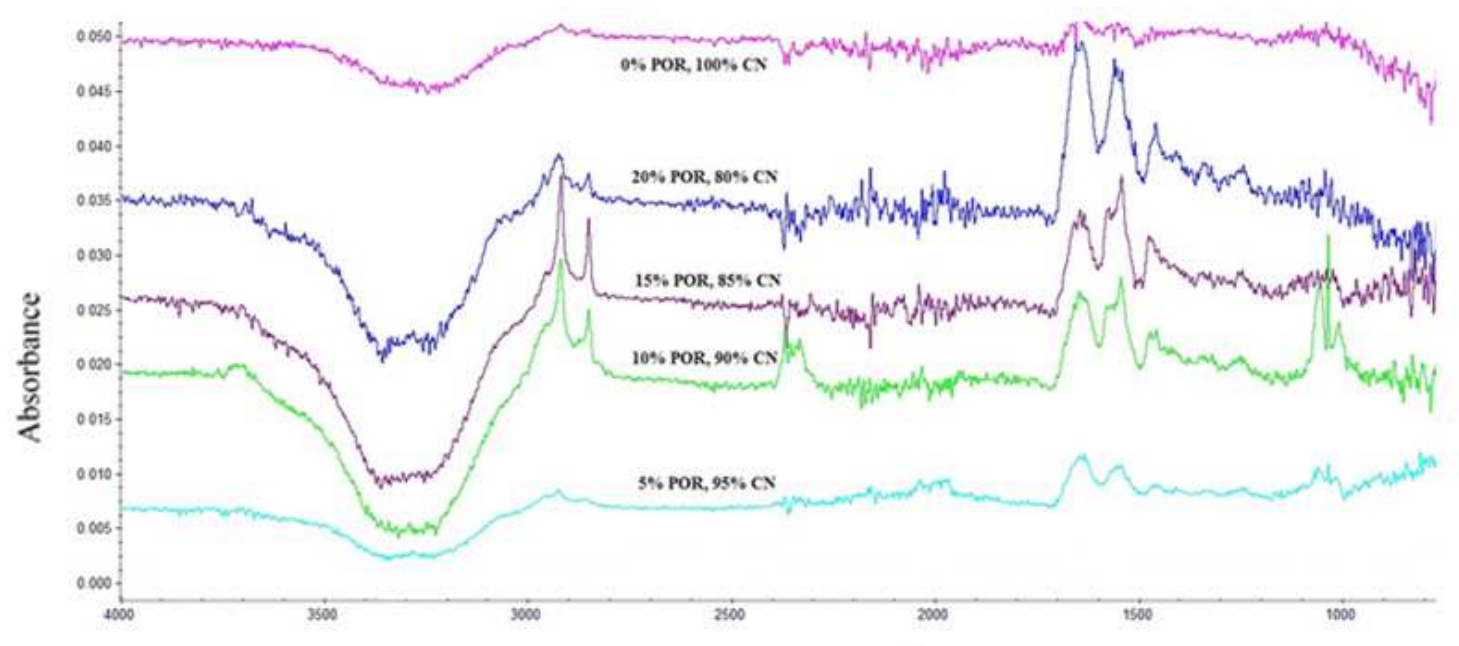

Wavenumber $\left(\mathrm{cm}^{-1}\right)$

(a)

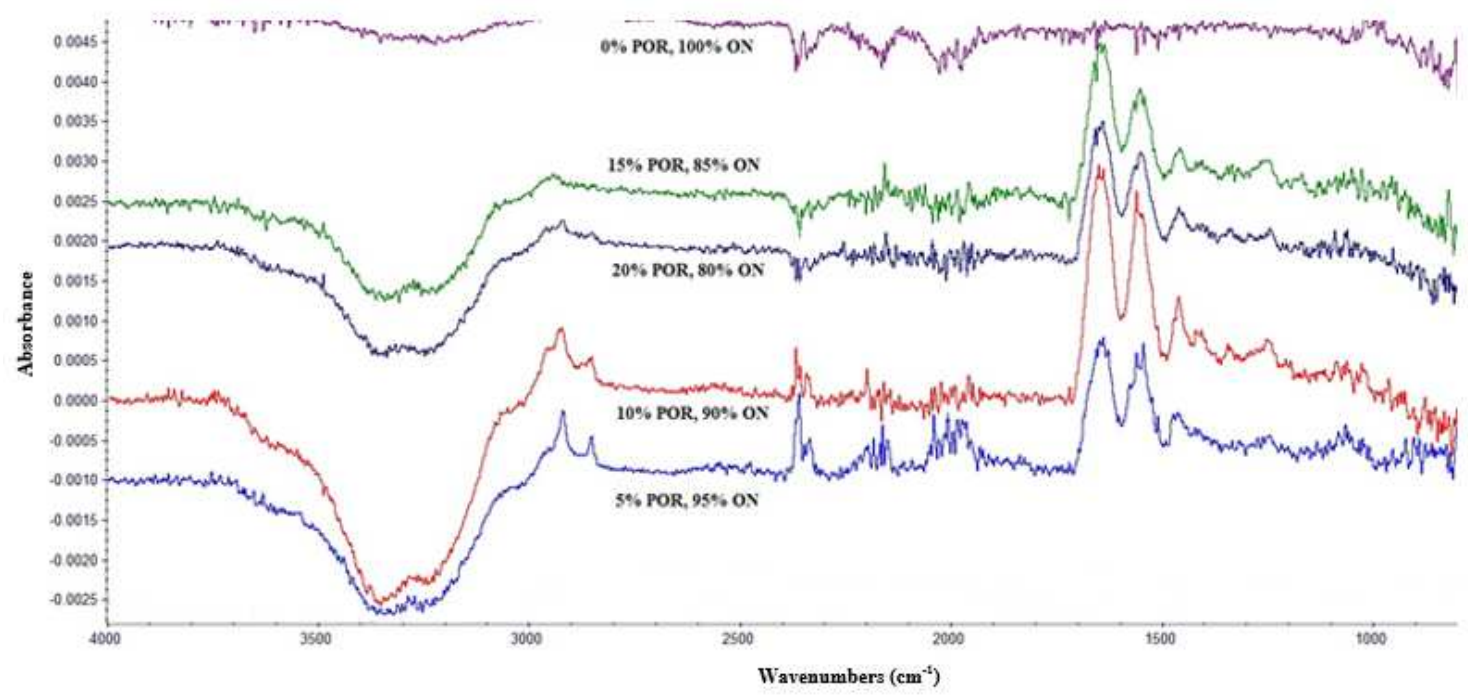

(b)

Fig. 3 FTIR spectra of spiked samples. (a) Cave nest adulterated with porcine gelatin. (b) Orange nest adulterated with porcine gelatin. Por: Porcine gelatin; CN: cave nest and; ON: orange nest. The numbers represent the percentage of adulteration. 


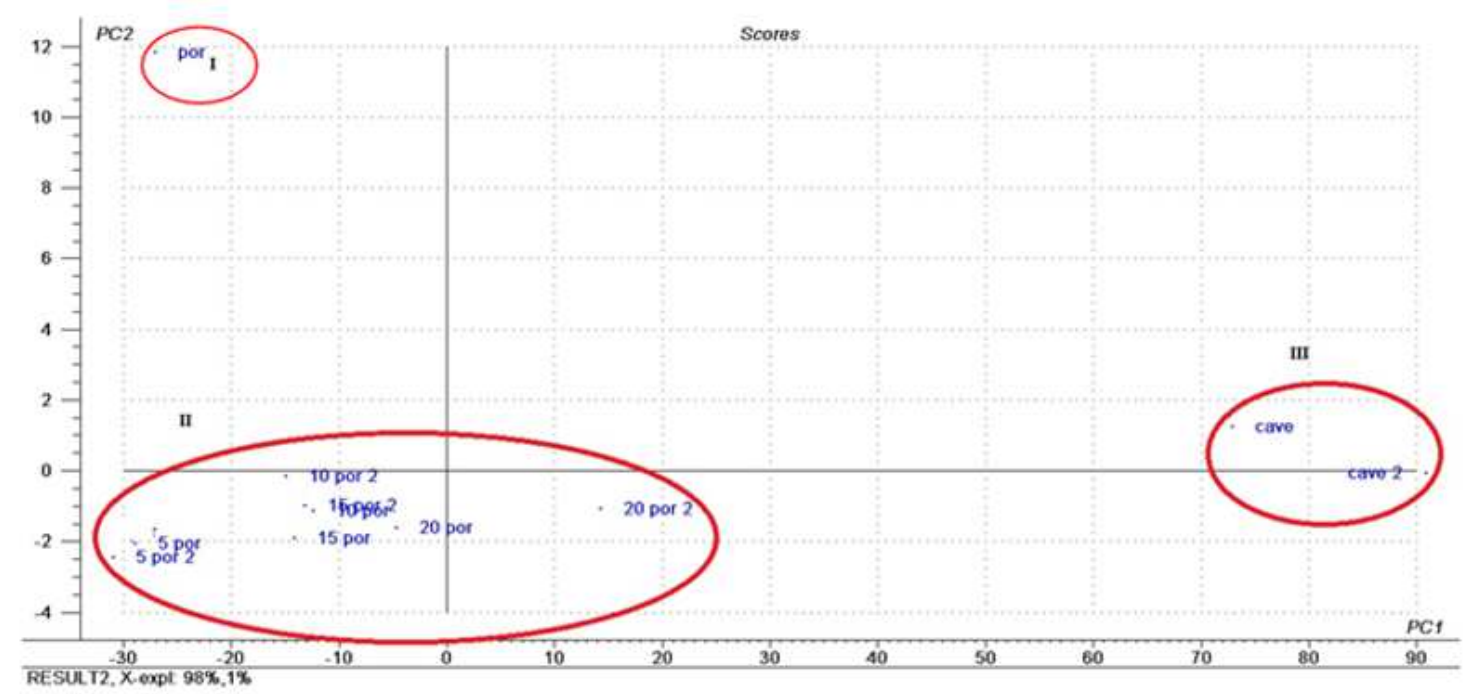

(a)

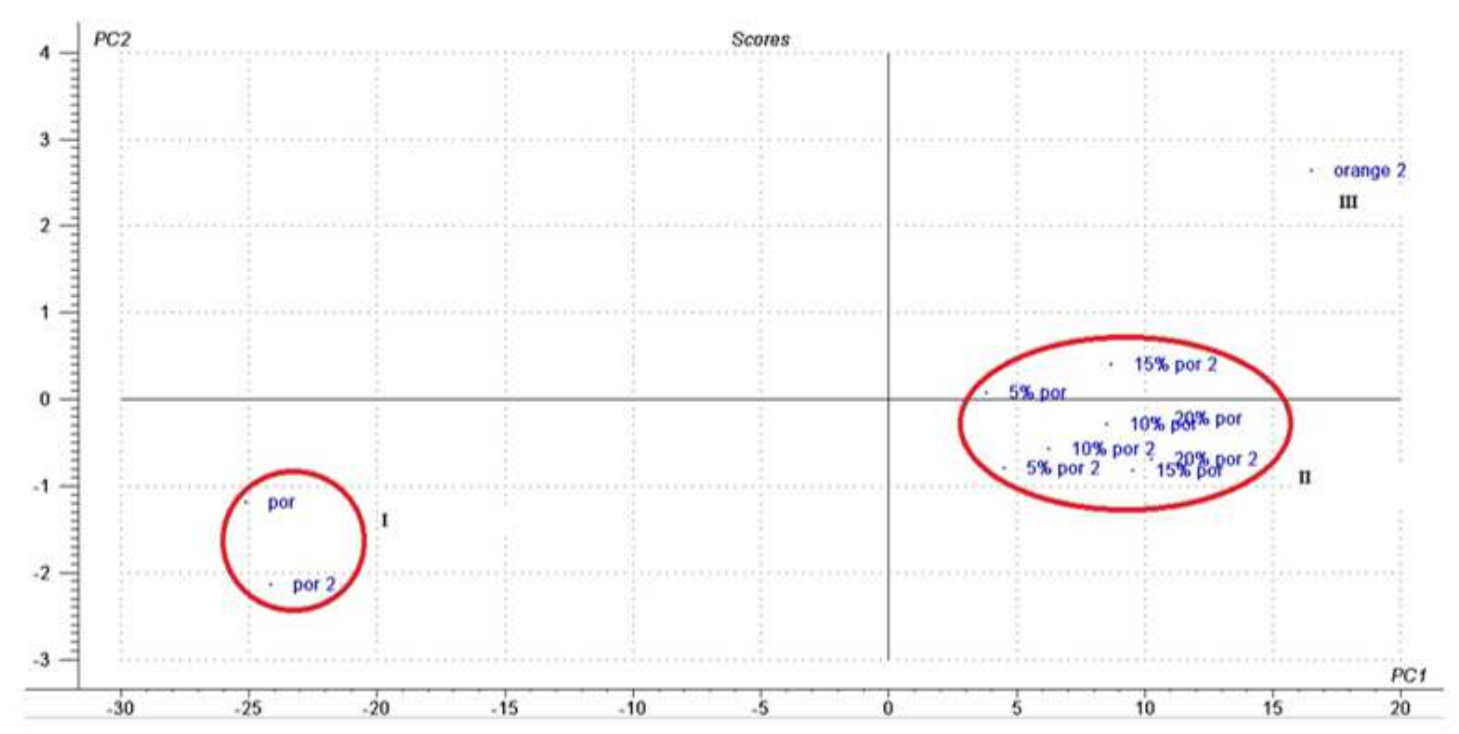

(b)

Fig. 4 Score plot of PCA classification. (a) Cave nests adulterated with different percentage of porcine gelatin. Group 1: porcine gelatin; group II: adulterated cave nests with porcine gelatin; and group III: cave nest. (b) Orange nests adulterated with different percentage of porcine gelatin. Group 1: porcine gelatin; group II: adulterated orange nests with porcine gelatin; and group III: orange nest. Por: Porcine gelatin; cave: cave nest; and orange: orange nest.

\section{Principal Component Analysis}

Principal Component Analysis (PCA) is one of the unsupervised multivariate analysis. This analysis allows data reduction to compress the number of uncorrelated variables into a smaller number of correlated variables designated as principle components (PC). It is commonly used to improve data interpretation and identified outlier of analytical methods [4],[13]. The outcomes are shown in loading and score plots. The evaluation of the scores enables the identification of sample class, while the evaluation of the corresponding loadings enables recognition of the related variables that contributed to the class of the samples. The association of numerous analytical methods such as Fourier transform infrared (FTIR) spectroscopy [10], [14], high performance liquid chromatography (HPLC) [15], visible and near-infrared (Vis/NIR) spectroscopy [16], matrixassisted laser desorption/ionisation time-of-flight (MALDITOF) [17] and gas chromatography (GC) [18] with PCA are generally employed to define the weak analytical differences using qualitative data.

1) Cave Nest Adulterated with Porcine Gelatin. The adulterated samples have been scattered into three groups (Fig. 3a). Porcine gelatin and unadulterated cave nests were located at the left (group I) and right (group III) side respectively. Cave nests that were added with $5-20 \%$ of porcine gelatin were located in the same group in the middle 
of groups I and III. For $98 \%$ and $1 \%$ of the variation, PC 1 and PC 2 were estimated; $99 \%$ of the variation was estimated for both PCs. Numbers in the score plot depict the percentage of the porcine gelatin in the samples. The results demonstrated that it is feasible to identify the cave nests that adulterated with $5 \%$ of porcine gelatin.

2) Orange Nest Adulterated with Porcine Gelatin. The adulterated samples were scattered into three groups (Fig. $3 b)$. Porcine gelatin and unadulterated orange nests were located at the left (group I) and right (group III) side respectively. Orange nests that were adulterated with 5 $20 \%$ of porcine gelatin were located in the same group in the middle of groups I and III. PC 1 and PC 2 estimated at 58\% and $15 \%$ of the variation, respectively; therefore, both PCs estimated $73 \%$ of the variation. The results show that it is possible to identifiy the orange nest that adulterated with $5 \%$ of porcine gelatin.

\section{CONCLUSIONS}

The FTIR technique was used to detect porcine gelatin in the processed EBNs. Porcine gelatin exhibited a dominant band at Amide I indicating the adulteration of EBNs with porcine gelatin. The FTIR spectra were analysed using PCA in order to identify the adulteration percentage in the samples. This method could detect at the minimum of $5 \%$ of porcine gelatin in EBNs in the assessment of the spiked samples. Future work on the detection of lower percentage of adulteration is required.

\section{ACKNOWLEDGMENT}

The authors are grateful for the financial support provided by the IIUM Research Initiative Grant Scheme (Project no.: RIGS16-068-0232) from International Islamic University Malaysia, Selangor, Malaysia.

\section{REFERENCES}

[1] J. R. Lin, Y. Dong, H. Zhou, and X. P. Lai, "Identification of Edible Bird's Nest by Electrophoresis," World Science and Technology/Modernization of Traditional Chinese Medicine 30-32, 2006.

[2] Y. Wu, Y. Chen, B. Wang, L. Bai, R. Wu, Y. Ge, and F. Yuan, "Application of SYBRgreen PCR and 2DGE methods to authenticate edible bird's nest food," Food Res Int. 2020-2026, 2010

[3] L. Guo, Y. Wu, M. Liu, B. Wang, Y. Ge, and Y. Chen, "Authentication of Edible Bird's nests by TaqMan-based real-time PCR," Food Control 220-226, 2014.
[4] A. M. Ismail, A. Aina, D. M. Hashim, and I. Amin, "Using amino acids composition combined with principle component analysis to differentiate house and cave bird's nest," Current Trends in Technology and Science 363-366, 2013.

[5] T. Nur Azira, I. Amin, M. Shuhaimi, and H. Muhajir, "Enzyme immunoassay for the detection of porcine gelatine in edible bird's nest," Food Addit Contam Part A 1023-1028, 2015.

[6] T. Nur Azira, I. Amin, M. Shuhaimi, and H. Muhajir, "Determination of porcine gelatin in edible bird's nest by competitive indirect ELISA based on anti-peptide polyclonal antibody," Food Control 561 - 566, 2016.

[7] N. Cebi, M. T. Yilmaz, and O. Sagdic, "A rapid ATR-FTIR spectroscopic method for detection of sibutramine adulteration in tea and coffee based on hierarchical cluster and principal component analyses," Food Chem 517-526, 2017.

[8] C. S. Whei Miaw, C. Assis, A. R. C. Sales Silva, M. L. Cunha, M. M. Sena, and S. V. C. de Sauza, "Determination of main fruits in adulterated nectars by ATR-FTIR spectroscopy combined with multivariate calibration and variable selection methods," Food Chem 272-280, 2018

[9] A. M. Jimenez-Carvelo, M. T. Osorio, A. Koidis, A. GonzalezCasado, and L. Cuadros-Rodriguez, "Chemometric classification and quantification of olive oil in blends with any edible vegetable oils using FTIR-ATR and Raman spectroscopy," LWT - Food Sci Technol 174-184, 2017.

[10] D. M. Hashim, Y. B. Che Man, R. Norakasha, M. Shuhaimi, Y. Salmah, and Z. A. Syahariza, "Potential use of Fourier transform infrared spectroscopy for differentiation of bovine and porcine gelatins," Food Chem 856 - 860, 2010.

[11] C. Ma, M. Rout, and W. Y. Mock, "Study of oat globulin conformation by Fourier transform infrared spectroscopy," J Agric Food Chem 3328 - 3344, 2001.

[12] R. M. Silverstein, F. X. Webster, and D. J. Kiemle, Spectrometric Identification of Organic Compounds United States of America: John Wiley \& Sons, Inc., 2005.

[13] B. Avula, T. J. Smillie, Y. Wang, J. Zweigenbaum, and A. Khan, "Authentication of true cinnamon (Cinnamon verum) utilising direct analysis in real time (DART)-QToF-MS," Food Addit Contam: Part A $1-8,2015$.

[14] M. D. Luca, W. Terouzi, G. Ioele, F. Kzaiber, A. Oussama, F. Oliverio, R. Tauler, and G. Ragno, "Derivative FTIR spectroscopy for cluster analysis and classification of morocco olive oils," Food Chem.," 1113 - 1118, 2011.

[15] M. Nemati, M. R. Oveisi, H, Abdollahi, and O. Sabzevari, "Differentiation of bovine and porcine gelatins using principal component analysis," J. Pharm. Biomed. Anal., 485-492, 2004.

[16] J. Gayo, and S. A. Hale, "Detection and quantification of species authenticity and adulteration in crabmeat using visible and nearinfrared spectroscopy," J Agric Food Chem., 585 - 592, 2007.

[17] A. Chambery, G. Monaco, A. D. Maro, and A. Parente, "Peptide fingerprint of high quality Campania White wines by MALDI-TOF mass spectrometry," Food Chem., 1283 - 1289, 2009.

[18] D. S. Lee, S. B. Noh, S. Y. Bae, and K. Kim, "Characterization of fatty acids composition in vegetable oils by gas chromatography and chemometrics," Anal Chim Acta., 163 - 175, 1998. 